\title{
Reminder Control System for Children Medical Care using Smart Phone Application
}

\author{
Faisal AlAwadhi', Abdulrahman Alkandari', Altaf Mishaal Alshammari ${ }^{3}$ \\ ${ }^{1}$ Electrical Power Department, High Institute of Energy, PAAET, Kuwait \\ fa.alawadhi@paaet.edu.kw \\ ${ }^{2}$ Computer Department, College of Basic Education, PAAET, Kuwait \\ aam.alkandariepaaet.edu.kw \\ ${ }^{3} \mathrm{Om}$ mabad middle school, Ministry of education, Kuwait \\ altaf.alshamri@hotmail.com
}

\begin{abstract}
The research project presents an iPhone application, which may assists in caring for infants. The application primarily helps parents to monitor their infant's vaccination schedules. In addition, the application is equipped with a medication manager and reminder when caring for a sick baby. Initially, the interfaces were designed using PicsArt application on iPhone. Then, the application was further developed using Xcode.

Keywords: reminder system, medical care system, baby development system, smartphone application.
\end{abstract}

\section{INTRODUCTION}

There are plenty of problems faced by parents in caring for their babies, especially first-time parents. Some of these problems include scheduling medications and determining adequate treatments for the baby. This paper addresses such problems and assists parents in understanding the needs of their children. The assistance allows parents to make informed decisions based on their babies' specific needs [1].

The increase of commitments and work-related stress among parents may result in the decline of attention towards newborn infants. This problem can be even more serious among mothers who have recently give birth. Therefore, it is the focus of this project to help mothers in such situations.

Capturing the needs of different babies, however, remains a major challenge. Due to practical constraint, our works mainly provide some of the most common guidelines for baby caring. For instance, in identifying suitable medications for various diseases among babies, we found plenty of medications for the same group of diseases. Thus, the selection of medications to be included in the application was performed manually rather than automatically.

This work describes Baby Planet, an iPhone application that serves as a reminder for newborn mothers. There are three main objectives of Baby Planet: to provide baby's developmental guidelines, to manage the scheduling of vaccinations and to keep track of medications for sick babies.

The application is initiated when a user entered the baby's information. The required information includes the baby's name, the date of birth and gender. Once the information is provided, the application starts to keep track of the babies developmental years.

Basic project requirements include iOS as the operating systems and Objective $C$ for development. Objective $C$ is an object-oriented programming language and was chosen for the project since it is the native language of the iOS platform. Other than that, the iOS software development kit (SDK) and cocoa library were used in the project. The use of Objective $C$ resulted in better performance compared to other programming language since Objective $C$ is the native language of the iOS.

As an object-oriented language, the code can be easily maintained and reused. The compilation process is also effortless with the Low Level Virtual Machine (LLVM) version 6.1.0. The LLVM provides optimization by converting the programmers' code into assembly code for the targeted platform.

\section{RELATED WORKS}

Kochan [2] demonstrated that the Xcode is an advanced tool that is easy to use and implement programs. This work clearly described the steps of application development using Xcode and the robustness of application built on the iOS platform.

Pridham and Chang [3] through the revised What Being the Parent of a New Baby Like (WPL) instrument suggested that soon-to-be parents examine their readiness by evaluating their perceptual performance and their adaptation to the paternal and maternal experiences.

Confavreux, Suissa, Saddier, Valérie Bourdès, and Vukusic [4] gave an insight into France's experience after introducing the vaccination program against hepatitis $B$ infection. Researchers tested the efficacy of the vaccination program and assess whether vaccination increased the risks of multiple sclerosis or resulted in relapse.

Chang, Zhu, Holroyd and K. Seng [5] suggested that the advancement of computing technology such as networked computers and smart mobile modem is capable for enhancing general health by delivering health related information to patients. In addition, the proliferation of mobile devices is helping accelerate e-health system. In the past, many developers have implemented e-health applications for portable devices such as Apple iPad and 
iPod since these devices provide rich visual experiences compared to other devices. A study on the top 200 mobile applications revealed a growing number of health applications indicating strong interests of developers in this area.

Snow [6] assessed the conversation between mothers and babies and found that simplicity of the speech was about the same at all ages, and none of the other features of the mothers' speech style showed any abrupt change at the time the children started to talk. The speech of two mothers to their infants at several points between three and eighteen months of age was analyzed. The changes that did occur started much earlier, at about seven months.

Finally, Morley [7] collected data on 28 symptoms and 47 signs from 1007 babies those under 6 months. Findings were systemically recorded for identifying the intensity of severe systemic illness system.

\section{BABY PLANET DESIGN}

The interface of Baby Planet is designed in such a way so that it is easy to use. The theme of the application is customizable depending on the gender of the baby. The icons at the bottom of the screen followed an informal approach in e-health applications. Baby Planet also provides a property to copy the information about a baby's development.

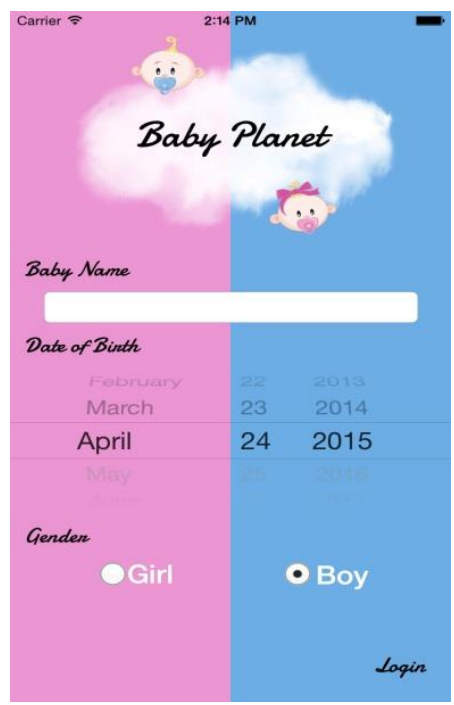

Figure 1 Login screen

As seen in Figure 1 above the screen included a text field, a date picker and radio buttons. The user should enter the baby's name in the text field (compulsory). Then, in the "datePicker", the user should enter the baby's birth date to calculate the baby's age. The "radio buttons" are to choose the gender of the baby. Selecting either girl or boy shall change the theme of the application.

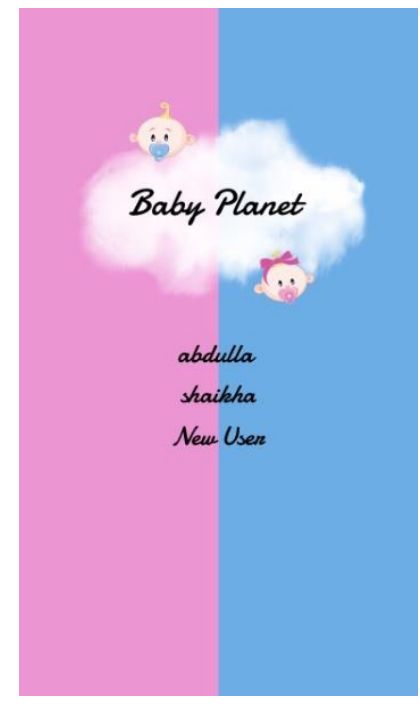

Figure 2 Account screen

Next, Figure 2 shows the first screen after the logo that shows the capability of the application to keep track of the development of multiple babies. Each of the babies has their own schedules and reminders. User may either opt to log out after saving the information, add a new baby or switch accounts.

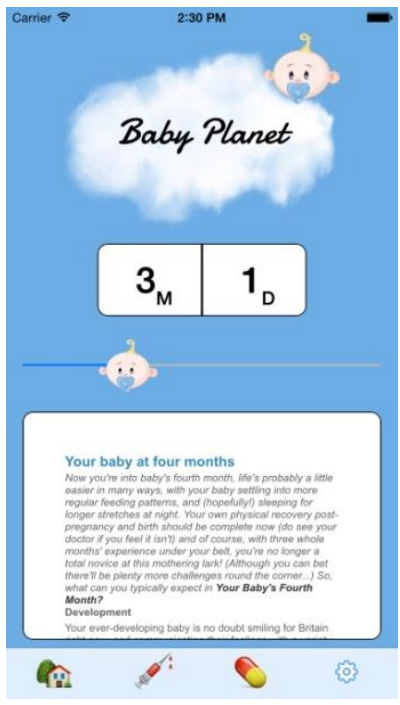

Figure 3 Home screen

Figure 3 above shows the Home screen which includes:

- The logo of the application.

- A Calendar view that captures the baby's age.

- An Age slider for added aesthetics

- A Details text view that describes the change of developmental growth of the baby. and

- A Tab bar with simple emoji for navigation.

User can view "Home" screen by clicking the "Home" icon. 


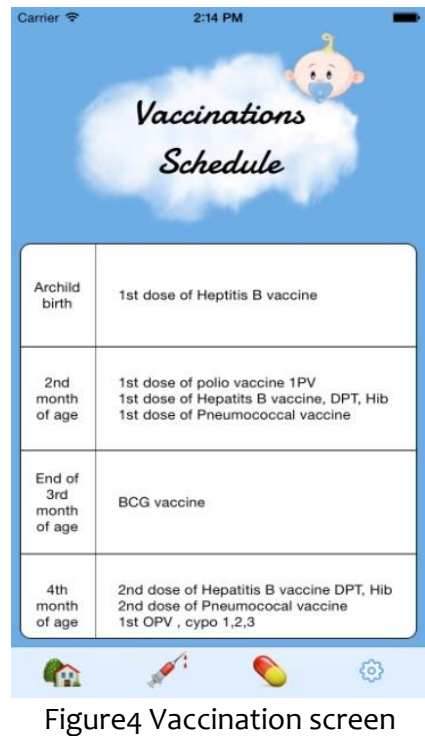

The "Vaccination" screen as shown in Figure 4, can be viewed by clicking on the syringe icon. It displays the vaccinations schedule for the baby up to one year of age. None of the information on this screen can be edited since it is simply intended to display the next vaccination appointment and to send reminders for the user.

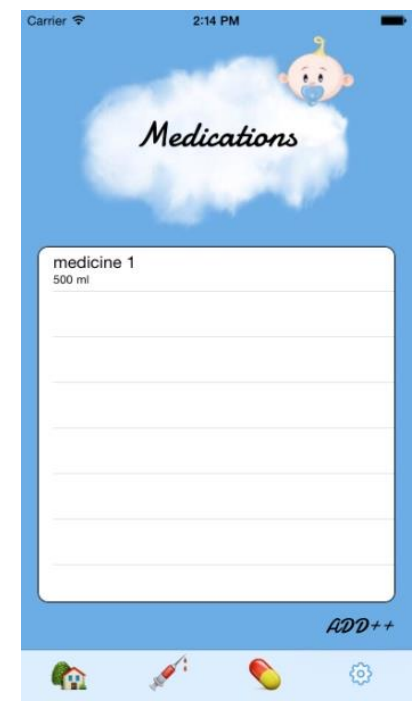

Figure 5 Medication screen

Figure 5 shows the "Medication" screen that displays a table for adding the name of the medication and the dose of the medication to be taken at any one time. The user may add the medication by tapping the "ADD++" button, and the user shall be brought to the "Add Medication" screen.

To return to the view the "Medication" screen, user may click on the medication icon on the tap bar.

This next screen (Figure 6) includes:

- Back button; to return to the previous screen.

- Three text-fields, the first field requires the user to enter the medication name, the second field requires entering the number of necessary days for taking the medication, the third field for the time allowed between the dosages.

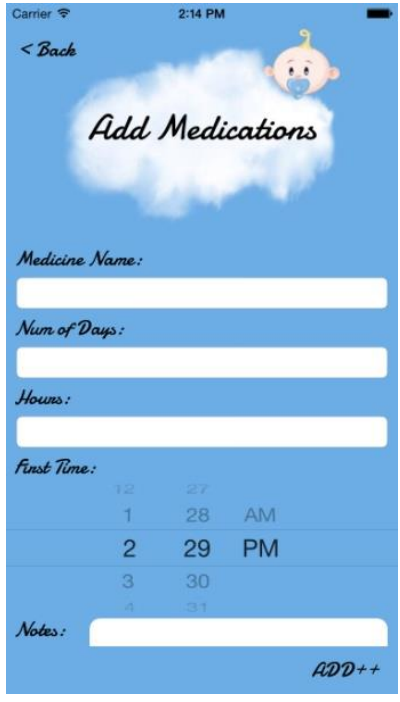

Figure 6 Add medication screen

- Time picker for setting the first time taking the medication.

- Text view for additional notes.

- Finally there is a button named "ADD++" to save all of the medication information.

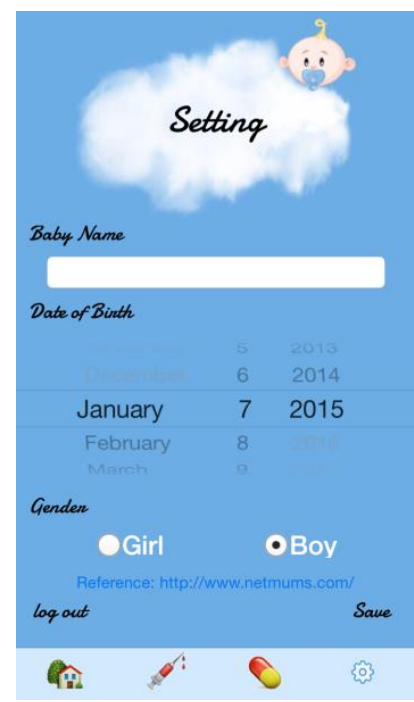

Figure 7 Setting screen

By clicking on the settings icon, the user will be brought to the "Settings" screen (Figure 7). This screen is similar to the login screen. User can edit the baby information (name, birthdate and gender). After changing the information, user has to click on the "Save" button to save all changes. There is also a "log out" button which brings the user to the initial screen (account screen).

Finally, there is an "Active" link added to this screen for retrieving information and advices about caring of babies.

\section{IMPLEMENTATION}

Initially, the authors gleaned the Apple developer's website to gain information about the program, the compiler and the recommended language for iOS application development. This leads to the use of Xcode and Objective $C$ programming language. Implementation 
works started immediately after downloading the recommended programs.

TABLE 1

GANTT CHART FOR BABY PLANET

\begin{tabular}{|c|c|c|c|c|c|c|c|c|}
\hline Phases & weeks & 1 & 2 & 3 & 4 & 5 & 6 & 7 \\
\hline \multicolumn{9}{|c|}{ Proposal } \\
\hline \multicolumn{9}{|c|}{ Definition } \\
\hline \multicolumn{9}{|c|}{ Concept } \\
\hline \multicolumn{9}{|l|}{ Design } \\
\hline \multicolumn{9}{|c|}{ Technical implemental } \\
\hline \multicolumn{9}{|l|}{ Testing } \\
\hline Evaluati & & & & & & & & \\
\hline
\end{tabular}

Table 1 shows the Gantt chart for the Baby Planet application. The main idea was presented during the first week followed by problem definition in the second week. Next, a few concepts that assists in the implementation of the project were added during the third week.

In the fourth week, a number of designs were presented prior to deciding on the best solution for the application. In the fifth and sixth week, programming and implementing took place. Each of the features was later tested in the seventh week to ensure its completeness. In the final week, the application was evaluated to ensure that it has met all objectives.

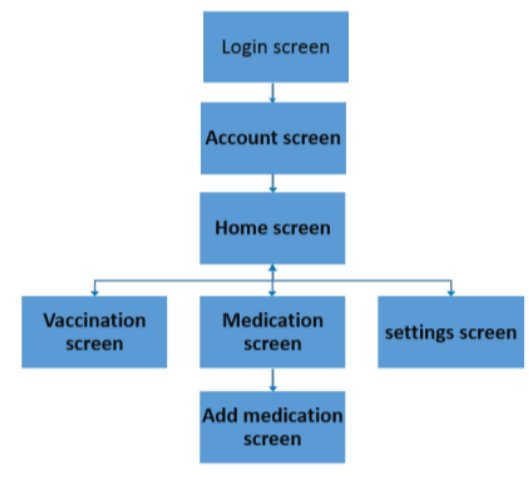

Figure 8 "Baby Planet" flow chart

Figure 8 describes the Baby Planet using a flow chart. The first screen is the login screen followed by an account screen and a home screen.

Home screen shows information about baby development, with further navigation to other submodules namely vaccination, medication and settings. Vaccination screen shows the vaccination schedule for the baby. Medication screen contains medication reminders, equipped with an "ADD++" button that brings the user to the "Add medication screen". The "Add medication" screen allows user to add a new medication reminder. The final screen is the settings screen that allow user to edit the baby's information.

Figure 9 shows the "login" flow chart that captures the flow of activities and responses when interacting with the application. The user must enter the baby's name, date of birth and select the gender.

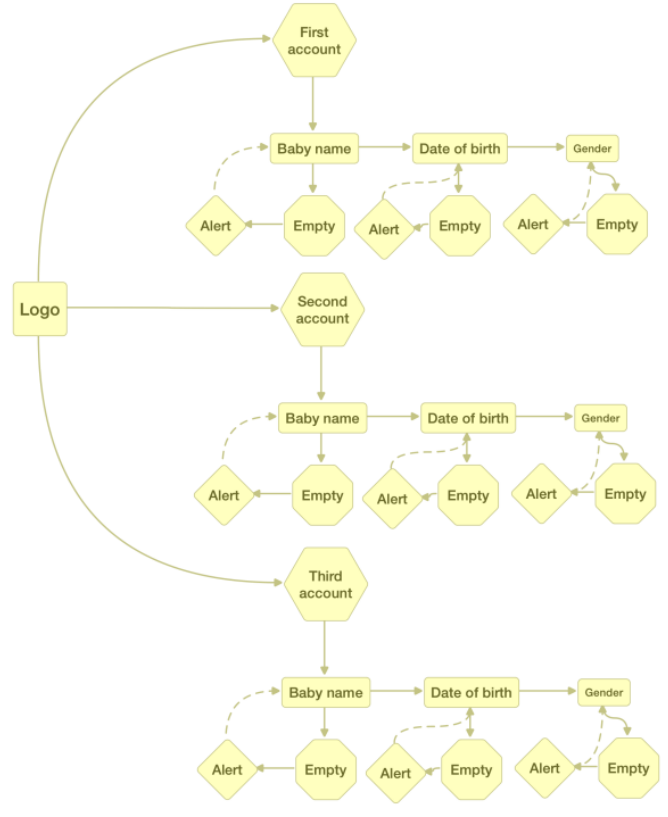

Figure 9 Login flowchart

All of this information are compulsory. If any of the textbox is empty, the application responded by sending an alert to complete the required information.

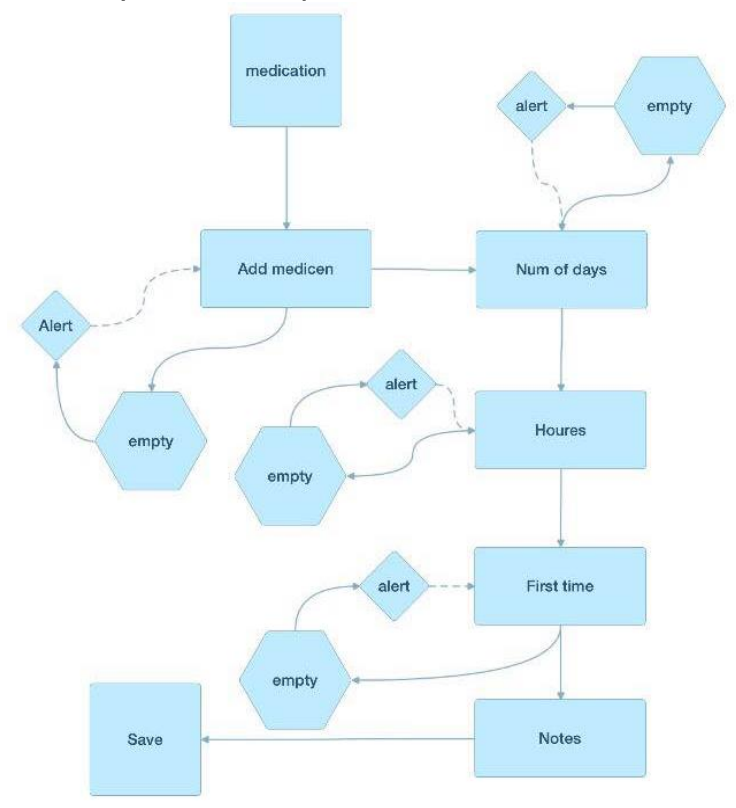

Figure 10 "Add Medication" flow chart

In Figure 10 the flow chart explains the "Add Medication" screen.

The screen requests the user to enter the name of the medication how many days does the baby needs the medication, the hours between the dosages, the first time taking the medication and additional notes. All of these fields are compulsory except for the notes. Similarly, the application sends an alert if any of the compulsory fields were empty. In the end, user clicks "ADD++" button to save the medication. 


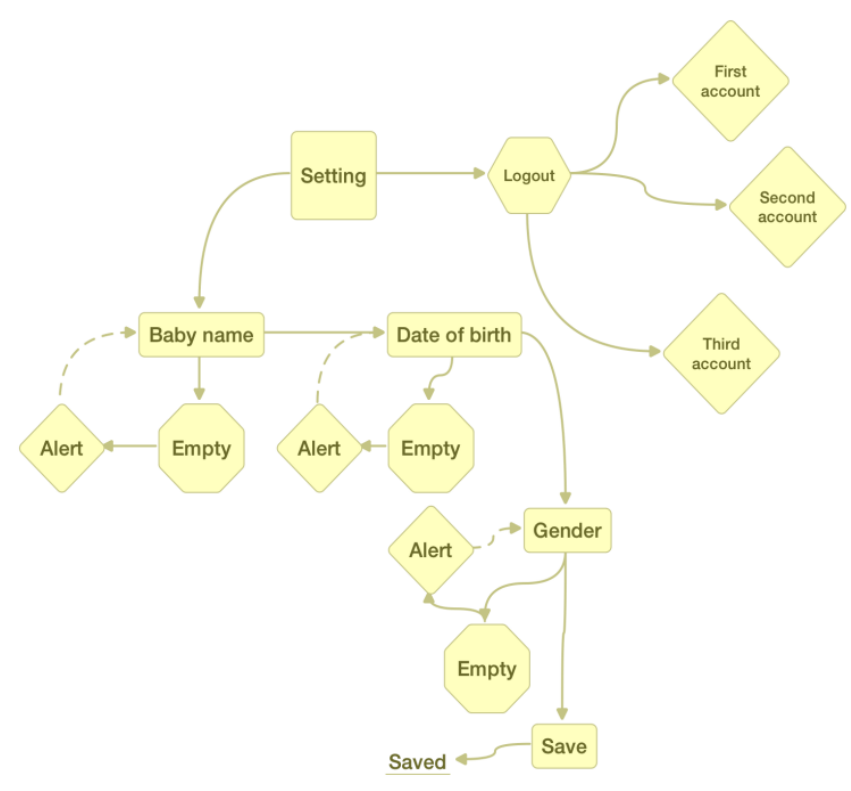

Figure 11 "Settings" flow chart

The flow chart shown in Figure 11 explains the "Settings" screen. In this screen, user may either logout from the account, edit baby's name, baby's birth date and baby's gender. All the fields are compulsory, and the system sends an alert if any of the fields was incomplete. User needs to click on the "Save" button to save the changes.

\section{CONCLUSION AND FUTURE WORK}

This paper presented an iPhone application named Baby Planet. Baby Planet is a very useful application that helps parents keep track of their baby's age and growth up to one year of age. The application provides a schedule for vaccinations and reminders for taking medication.

In the future, the application can be enhanced to support multi-languages to serve other user segments around the world. In addition, tracking of the baby's developmental growth shall be extended for more than one year. More information about the baby can be added such as the baby's current length and weight which can be further linked to pediatrician's contact information in the case of abnormalities.

\section{REFERENCES}

Association of Childrens Welfare Agencies and NSW Family Services Inc Developing Practice: The Child, Youth and Family Work Journal, Issue 23 (Autumn/Winter 2009).

S. G. Kochan. Programming in Objective-C. Addison-Wesley Professional, 2011.

Pridham, K F., and Audrey S. C. "What being the parent of a new baby is like: Revision of an instrument." Research in nursing \& health, vol. 12(5) (1989), pp. 323-329.

C. Confavreux, S, Suissa, P., Saddier, V, Bourdès, and S. Vukusic. (2001). "Vaccinations and the risk of relapse in multiple sclerosis". New England Journal of Medicine, vol. 344(5), pp. 319-326.

L. Chang, Q. Zhu, K. A. Holroyd, and E. K. Seng. "Status and trends of mobile-health applications for iOS devices: A developer's perspective." Journal of Systems and Software, vol. 84 (11) (2011), pp. 2022-2033.

C. E. Snow. "The development of conversation between mothers and babies", Journal of Child Language, vol 4(1) (February 1977), pp 1-22.

C. J. Morley, et al. "Baby Check: a scoring system to grade the severity of acute systemic illness in babies under 6 months old", Archives of disease in childhood, vol. 66(1), (1991), pp. 100-10. 